\title{
ONTONOTES: A UNIFIED RELATIONAL SEMANTIC REPRESENTATION
}

\author{
SAMEER S. PRADHAN \\ $B B N$ Technologies, Cambridge, MA 02138, USA \\ pradhan@bbn.com \\ EDUARD HOVY \\ ISI/USC, Marina Del Rey, CA 90292, USA \\ hovy@isi.edu \\ MITCH MARCUS \\ University of Pennsylvania, Philadelphia, PA 19104, USA \\ mitch@cis.upenn.edu \\ MARTHA PALMER \\ University of Colorado, Boulder, CO 80309, USA \\ palmer@colorado.edu \\ LANCE RAMSHAW* ${ }^{*}$ and RALPH WEISCHEDEL ${ }^{\dagger}$ \\ $B B N$ Technologies, Cambridge, MA 02138, USA \\ *lramshaw@bbn.com \\ †weischedel@bbn.com
}

\begin{abstract}
The OntoNotes project is creating a corpus of large-scale, accurate, and integrated annotation of multiple levels of the shallow semantic structure in text. Such rich, integrated annotation covering many levels will allow for richer, cross-level models enabling significantly better automatic semantic analysis. At the same time, it demands a robust, efficient, scalable mechanism for storing and accessing these complex inter-dependent annotations. We describe a relational database representation that captures both the inter- and intra-layer dependencies and provide details of an object-oriented API for efficient, multi-tiered access to this data.
\end{abstract}

Keywords: OntoNotes; shallow semantics; annotation.

\section{Introduction}

The OntoNotes project is addressing the challenge of large-scale, accurate, and integrated annotation of multiple levels of the shallow semantic structure in text. Experience has shown that when individual levels like syntactic parse structure, propositional structure, and semantic role labels can be annotated with high consistency (inter-tagger agreement, or ITA), then machine learning models can also 
be implemented to predict those structures with only somewhat lower accuracy. The premise of OntoNotes is that integrated annotation covering many levels will allow for richer, cross-level models enabling significantly better automatic semantic analysis.

Given that goal, the mechanism used to store and access the annotation becomes a key part of the research, and not just a tool support issue. The representation has to correctly capture the dependencies between the different annotation layers, so that consistency can be maintained across layers. The representation also has to allow for unified access, so that cross-layer features can be used in the integrated predictive models that will make use of these annotations. This paper describes how we have addressed the research challenge of modeling such multi-layer annotations, with complex, cross-layer dependencies, while providing efficient, convenient, integrated access to the data.

\section{OntoNotes}

The OntoNotes project [7] is a multi-year, collaborative effort between BBN Technologies, the University of Colorado, the University of Pennsylvania, and the University of Southern California's Information Sciences Institute.

\subsection{Data}

OntoNotes 2.0 consists of newswire and broadcast news data from two languages English and Chinese. The English portion is a $300 \mathrm{k}$ word, approximately 600 documents collection from the non-financial news portion of the Wall Street Journal (WSJ), and about $200 \mathrm{k}$ word, approximately 950 documents from a collection of broadcast news sources such as CNN, ABC, NBC, etc. The Chinese newswire portion is a $250 \mathrm{k}$ word collection comprising 325 documents from the Xinhua and about 80 documents from the Sinorama magazine. The broadcast news portion comprises about $300 \mathrm{k}$ word, approximately 1200 documents from news sources such as CBS, CNR, CTV, etc. These documents are annotated with the following layers of information:

(1) Syntax - A syntactic layer representing a revised Penn Treebank $[1,10]$.

(2) Propositions - The proposition structure of both verbs and nouns in the form of a revised PropBank $[1,13]$.

(3) Word Senses - Coarse grained word senses are tagged for the most frequent polysemous verbs and nouns, in order to maximize coverage. The word sense granularity is tailored to achieve $90 \%$ inter-annotator agreement as demonstrated by Palmer et al. [12].

(4) Names - The corpus was tagged with a set of 18 proper name entity types that were well-defined and well-tested for inter-annotator agreement by Weischedel and Brunstein [19].

(5) Coreference - General anaphoric coreference that spans a rich set of entities and events - not restricted to a few types, as has been characteristic of most 
coreference data available until now - has been tagged with a high degree of consistency. Attributive coreference is tagged separately from the more common identity coreference [16].

(6) Ontology - The Ontology is a refinement over the Omega ontology [14] and is composed of an upper model - which is a network of concepts, and a collection of sense pools which identify more fine-grained notions in the meaning space. Each sense pool represents a manually selected collection of synonymous OntoNotes senses of different words, which are then connected to the relevant concepts in the upper model. The creation and verification of the sense pools is described in $\mathrm{Yu}$ et al. [20].

For a more detailed description of the different layers, the reader is referred to Hovy et al. [7].

\subsection{The challenge}

To the best of our knowledge, this is the first time that an attempt has been made to integrate so many different, rich, layers of syntax and semantics. Although, in combination, we had decades of experience in annotating the individual layers (excluding coarse grained word sense, and general anaphoric coreference), we had no mechanism for integrating them. This posed a significant challenge as it is more or less an open problem, and there exist no off-the-shelf resources that can be used to meet this end.

The questions that this level of integration pose are:

(1) How do we ensure that all the components are consistent with each other? The types of inconsistencies that we encounter in this process are two fold: (i) Those that challenge the underlying assumptions and mechanics of the annotation differences and require a careful revision of the inter-connecting components, and (ii) Those that are more technical (engineering/formatting) in nature. Although there is no silver bullet for solving either one, a solution to the latter can significantly alleviate problems encountered during representational manipulations.

(2) How do we distribute all these different layers of data? Should we distribute them as independent pieces and leave the task of assembling them to the end user or, do we provide an integrated representation that greatly simplifies the dissemination of this rich information? If the latter, what would be the best way to accomplish this?

(3) What type of representation would best facilitate the use of this information as training data for systems that will be incorporated into applications with their own knowledge sources? Can this representation also support leveraging these additional knowledge sources during the training process?

\subsection{Properties of an ideal solution}

Since these layers represent related linguistic information, there is a high degree of interconnection between them. To begin with, the PropBank annotations are 
defined over nodes in the Treebank. Most ${ }^{\mathrm{a}}$ of the coreference links have also been defined, by design, over the nodes in the Treebank. Vital information relating to the interpretation of each word sense, including its relation to the WordNet senses, their argument structure, as well as the constraints imposed on arguments by the particular semantic frame that a predicate invokes, are captured in the sense inventory and frame files. In short, the information required to interpret the semantics of a sentence is spread over several different files, and without some mechanism to control this information, it can easily become asynchronous.

An ideal solution would be one that combines this information in an integral whole which allows an end-user to both easily interpret all the vital connections as well as to easily manipulate the information. The representation of such layers, we believe, should provide a bare-bones structure independent of the individual semantics with the following properties:

(1) Efficiency - It should efficiently capture both intra- and inter-layer semantics

(2) Independence - It should maintain the independence of each annotation layer. Any component should be replaceable with a parallel representation. For example, it should allow replacing PropBank with FrameNet [3]. That is, as long as the parallel representation exhibits the same core properties, it should be easily incorporable into the whole.

(3) Flexibility - It should provide mechanisms for flexible integration. For example, it should accommodate a change in representation of, say, propositions over spans of text instead of over nodes of a given syntactic representation.

(4) Granularity - It should be integrated at a level of granularity so as to allow relatively easy integration of more components.

(5) Robustness - It should capture first-order, or primary connections between the components in such a way that secondary connections do not become inconsistent upon a superficial change in representations.

(6) Queriability - It should facilitate cross-layer queries.

(7) Versioning - It should not be limited to storing the annotations themselves, but should manage different versions and hypotheses generated by automatic systems, allowing them all to coexist.

\section{A Solution}

We have developed a representation which we believe possesses all the above qualities to a more or less satisfactory degree: a relational database representation that is used to define and store the required semantics underlying the data, and an object layer which allows for intuitive manipulation of this data.

\footnotetext{
a Some entities/events constitute subparts of the relatively flat NP structure in the Treebank and have to be defined over word spans instead of corresponding to nodes.
} 


\subsection{Relational layer}

The main reason for using a relational database representation is that it allows for flexibility of design and provides an objective language to efficiently specify all the interconnections in the form of its schema. Furthermore, there exist timetested mechanisms to deal with the problem of maintaining consistent states of each layer, and at the same time allow for concurrent modifications to individual layers. In short, this is done by defining dependencies through the use of Primary and Foreign keys over database tables. The reader can find this information discussed in depth in any standard text book on the subject of relational databases.

The entity relationship diagram for the database is shown in Fig. 1. The tables shown are divided into six logical blocks depending on the type of linguistic

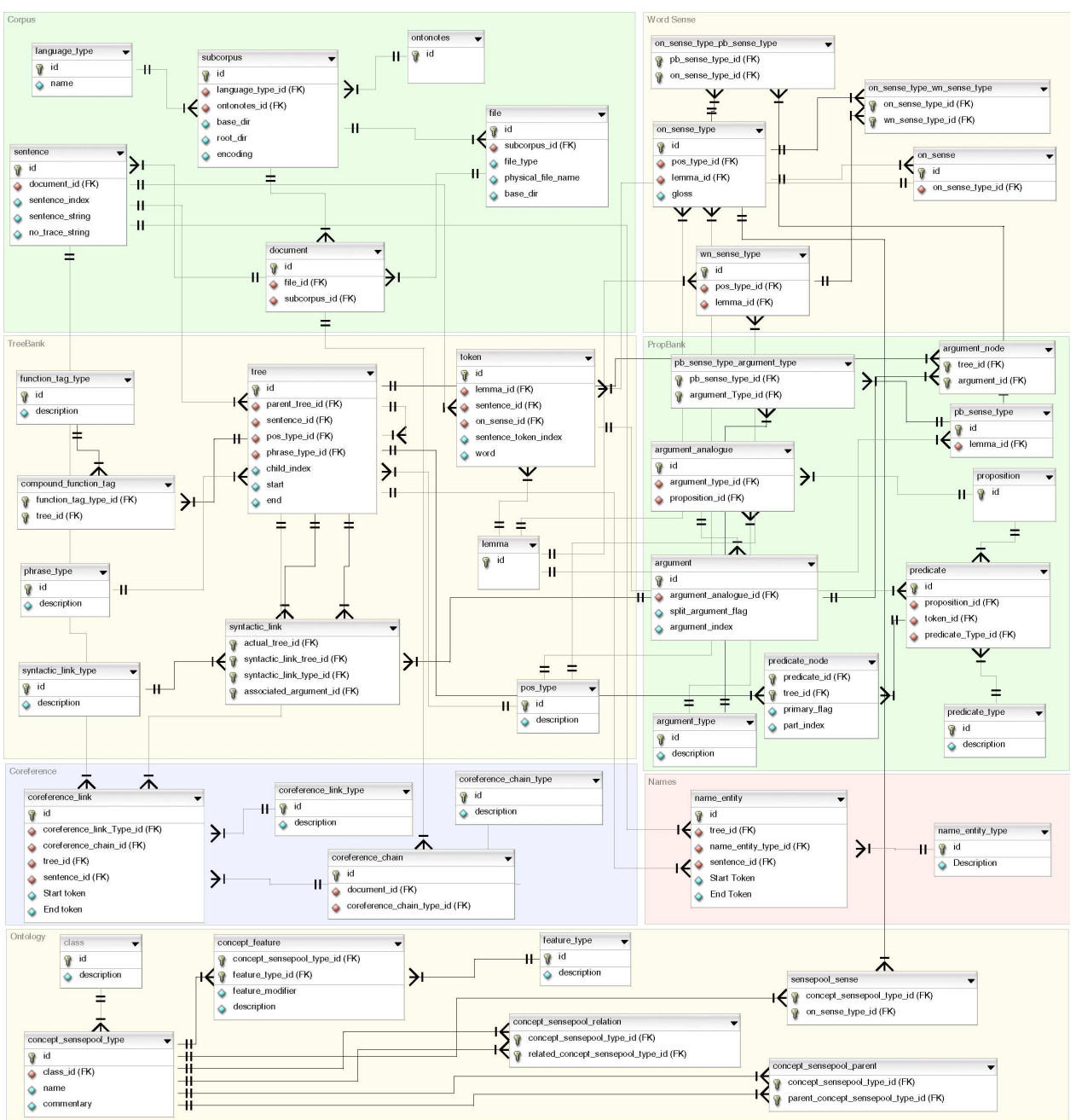

Fig. 1. Entity-relationship diagram of the OntoNotes database. 
annotation that they represent: (i) The corpus itself, (ii) Treebank, (iii) PropBank, (iv) Word Sense, (v) Names, (vi) Coreference and (vii) Ontology. Each of the annotation schemes adds meta-information to the corpus. The lowest common granularity of annotation is represented by the Treebank tokens, ${ }^{\mathrm{b}}$ rather than the more prevalent character-based indexing of several existing corpora, significantly simplifying the visualization and manipulability of the data. If required, the token-based indexing scheme allows for dereferencing to lower levels of atomicity without affecting the interface between the corpus and other layers of annotation. This could be achieved by the addition of a table that maps to the lower level, thus providing a comfortable degree of encapsulation. This tokenization is particularly well-suited for the existing layers of annotation as they take the Treebank tokenization as a basis of dereference. There are a few exceptions; for example, in the case of coreference for about $2 \%$ of the cases an entity is (most likely) a subpart of one of the flat-NPs that the Treebank realizes. This requires only the addition of a derivable layer of token-based offsets. In the case of names, although the original annotation is based on token-offsets, about $93 \%$ of the names actually align with the existing nodes in Treebank. In such an instance, we provide a derived layer of nodes corresponding to the named entities. In short, the database captures the primary level of granularity and allows for the flexibility of adding derived layers for better interpretation when necessary.

In reviewing the design, note that all the tables with the suffix _type indicate tables that only store type information. The others represent instance tables where instances, instead of just the type information, are stored. Various cardinalities such as one-to-one or one-to-many relationships are shown using the crow's feet style of representation. The ontonotes table contains the id for the OntoNotes corpus. This is associated with the many subcorpora that represent it - identified by the subcorpus table. The subcorpus contains many files captured in the file table, which in turn contain one or more documents (the document table). The document then contains one or more sentences (the sentence table). This now puts a structure on the raw text which we are embellishing with layers of linguistic information. The second logical block is the Treebank. Here at the center lies the tree table which represents a general case of trees, nodes and leaves. The root tree has a NULL parent whereas the leaves have NULL children. Hierarchical information is captured through table recursion. The meta-information on the tree nodes is captured in the compound_function_tag and function_tag_type tables. There can be more than one function tag associated with a node in the tree, and many nodes associated with the same function tag type - known as a many-to-many relationship in database terms, and the design principles dictate the generation of a link table which we call the compound_function_tag table and which contains a composite primary key which is made up of two foreign keys: one being the primary key of the function_tag_type

${ }^{\mathrm{b}}$ Words tagged with part of speech in the Treebank. 
table; and the other being the primary key of the tree table. For the sake of generality, each tree has an associated token and other syntactic information such as the part of speech type (pos_type table), the phrase type (phrase_type table), the trace chain (syntactic_link table). The reason for giving the latter table a more general name is that it is also used to capture links from the propositions as indicated by the reference to the argument_id.

The PropBank logical block captures the propositional annotation. Here, at the center lies the proposition table which has associated with it a predicate and one or more arguments. Both the predicate as well as the arguments exhibit a many-to-many relationship with the nodes in the Treebank. Therefore, we create two more link tables predicate_node and argument_node. predicate_type and argument_type tables capture the respective type information. Each predicate in the PropBank invokes a semantic frame and that determines which of its core arguments such as ARG0, ArG1, etc. can be legally associated with that predicate as well as the semantics of those arguments. This frame type is represented in the pb_sense_type table, and the pb_sense_type_argument_type serves as the link table. This in turn is connected across the logical boundary to the on_sense_type_pb_sense_type table in the Word Sense portion of the database. What this means is that the OntoNotes sense type has a many-to-many mapping to the PropBank frame sense. A similar relationship to the WordNet senses is captured in the wn_sense_type and on_sense_type_wn_sense_type and the wn_sense_type tables.

Names are represented with the name_entity and name_entity_type tables in the logical block called Names, and the tables coreference_chain and coreference_link capture the coreference chains and links within each document in the corpus. The coreference_chain_type and coreference_link_type store the respective type information. As mentioned earlier, most of the coreference links and names correspond to a node in the tree, and that information is stored as tree id in the respective table where applicable, or NULL otherwise.

Finally, the ontology is represented within the Ontology logical block. In the database schema, the concept_sensepool_type table represents the concepts and sense pool types. At some fundamental level, the concept and sense pool represent points in the meaning space, therefore, in order to avoid separation of similar logic, and thereby fragmentation of tables in the database, we choose to create one composite table for both the entity types and use the class attribute to distinguish between the two. Each concept and sense pool can have multiple parents as well as children. Furthermore, each concept and sense pool can potentially exhibit a similarity that does not justify hierarchical relationship, or membership, and these similarities are captured using a "related" link. Since these interconnections exhibit a many-to-many property, the obvious choice in the relational world is to create link tables, which are the concept_sensepool_relation and concept_sensepool_parent table respectively. One more thing that remains is the connection of the ontology to the OntoNotes word sense portion which is captured in the sensepool_sense table which contains pointers to the individual OntoNotes senses that comprise the sense 
pool. There were a few stages in the design process where we could have imposed some hard constraints on the relations, such as - a concept can only have another concept, and not a sense pool, as it parent; or, imposed a mutual exclusion constraint on the membership of senses in a sense pool, but we refrained from adding such constraints since the OntoNotes ontology is still in developmental stage, and it might not be wise to back-propagate any such constraints from the physical data design to the conceptual level. We would like to let the refinement of the ontology generate such constrains, if necessary.

We used the MySQL database to realize the database design.

\subsection{Object layer}

A well-defined relational layer provides a clear foundation for designing the object layer. To facilitate a better design of the object layer, we took the following decisions:

(1) We decided to trade-off database normalization for database design elegance and integration with the object world. Therefore, we did not normalize the tables beyond the first normal form.

(2) We decided to create a composite primary key for each table rather than using auto-increment, or using a database generated composite primary key. The goal here was seamless integration between the relational and object world. This meant that the database primary keys can also be used to index the objects in the respective containers. Another advantage of this is that the keys by themselves have a lot of semantic value and a person looking at them can, to some extent, decipher what it represents.

In the object design, almost every database table corresponds to an object with the database columns representing part of the attributes. There is almost always more derived information required for logical manipulation that is part of an object which is not in the database table itself. This resonates with the original plan of having only the bare-minimum of information needed to represent the data in the database. If required, more complex logic can be easily built in the object layer. Also, more often it is the case that the objects inside container objects are skeletal representations of those objects thus maintaining component independence.

Depending on the interface requirements of a particular application, the level of detail in each object can be altered to meet various needs. As an example, the word sense of a lemma can be tagged to be "1" but that "1" actually represents the sense "1" which has various attributes associated with it, such as its definition, the associated PropBank frame groups, the associated WordNet sense groups, etc. This is achieved by just accessing the sense_inventory class which centralizes information for each lemma and their senses, mappings, etc. This has an alternative benefit of avoiding possible redundancy and therefore inconsistency. Figure 2 shows the UML representation of part of the package on.corpora which comprises, among others, the modules on.corpora.tree and on.corpora.proposition. The argument_part class 


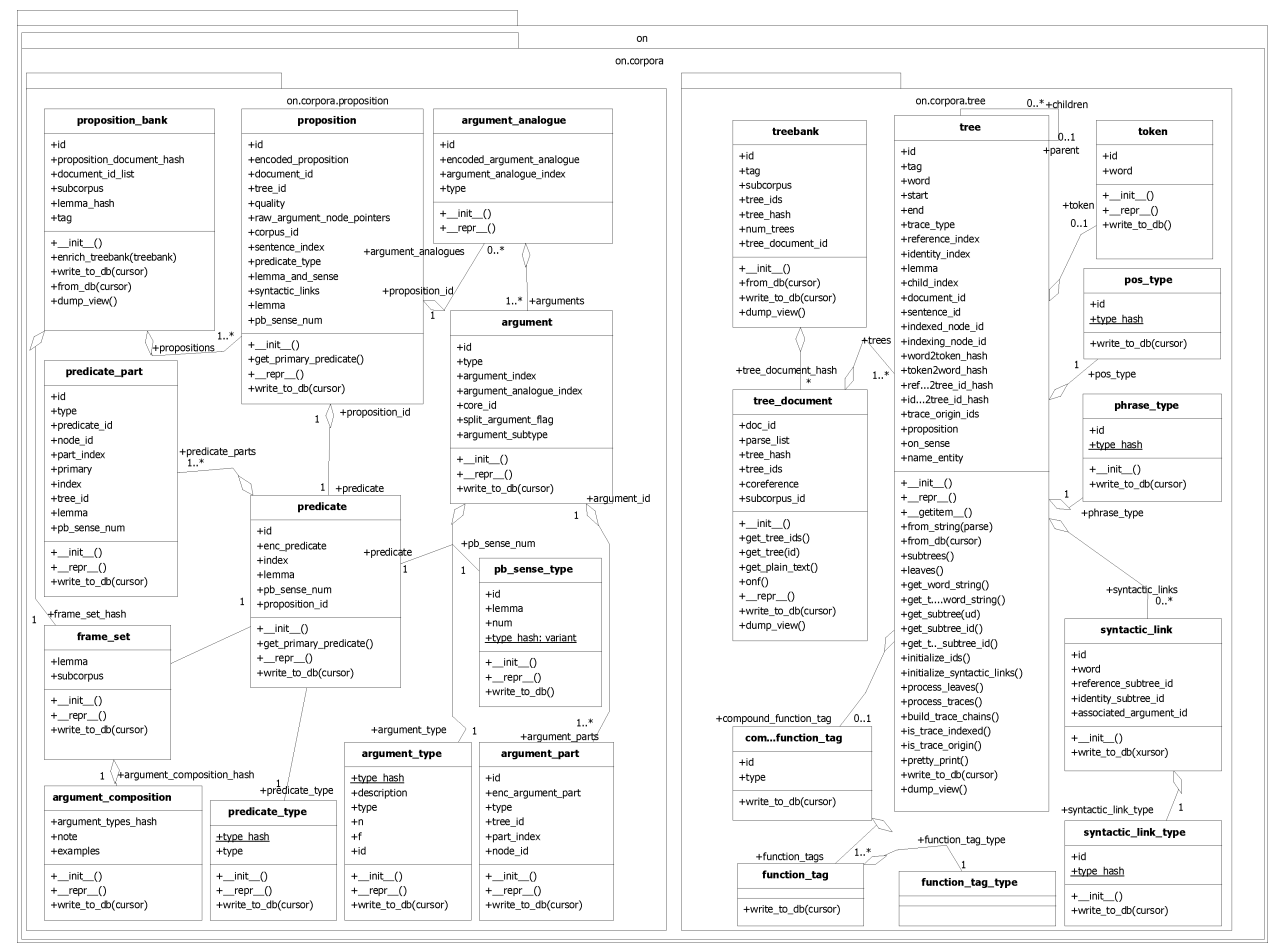

Fig. 2. UML representation of the modules on.corpora.tree and on.corpora.proposition which represents the Treebank and PropBank, respectively.

represents the information in the argument_node table. The argument_analogue represents the table with the same name in the database world, and it captures the fact that an argument can be represented by multiple nodes in the tree - a trace node, which points to possibly another trace node which points to the actual node that identifies the string that the argument is represented with. All three, in the PropBank world, are considered to be equivalents of the same argument. Therefore the name "argument_analogue". The attribute argument_analogue_index is in order to maintain the directional association between these equivalent arguments. Furthermore, each layer is provided with application level logic to integrate itself with the other layer that it has an integral connection with. For example, in this particular case, owing to the centrality of the Treebank the propbank and on sense classes have been provided with enrich_treebank methods which make the necessary connections, thus forming full-fledged objects. Whereas, name_bank, and coreference_bank have been provided with enrich_align_treebank methods that try to align the token spans with nodes in the tree when there exists one.

More details on the design can be found in the ontonotes-db-tool API that has been submitted for distribution with the corpora. The Python programming language has been used to implement the object layer. 


\section{Interaction Lifecycle}

We will take a brief look at a typical interaction between the raw data, database, and object layers. A few lines of code allow a pre-defined corpus file-structure to be read and converted into objects. The application logic could identify several errors and inconsistencies in the raw data that are encountered during object creation. These are mostly at the independent object level. Upon successful object creation they are written to the database. The constraints imposed by the data model in the database possibly identify some more intra or inter-layer inconsistencies ensuring that the data that gets stored in the database is clean and consistent. Figure 3 graphically depicts this cycle. Each object uses its write_to_db(cursor) method to write itself to the database. The top-level container contains the overall logic whereas the intermediate containers know how to write themselves to the database and these methods are delegated from top down to achieve the desired result. Once the data is in the database the ontonotes-db-tool API can then be used to read in the database, and generate the objects from it. All top level containers know how to re-create the full-fledged objects and pointers to objects as required. This is possible through the from_db(cursor) method.

In a typical database initialization lifecycle, the raw data will be read and written to the database iteratively until all the errors and inconsistencies are solved. When

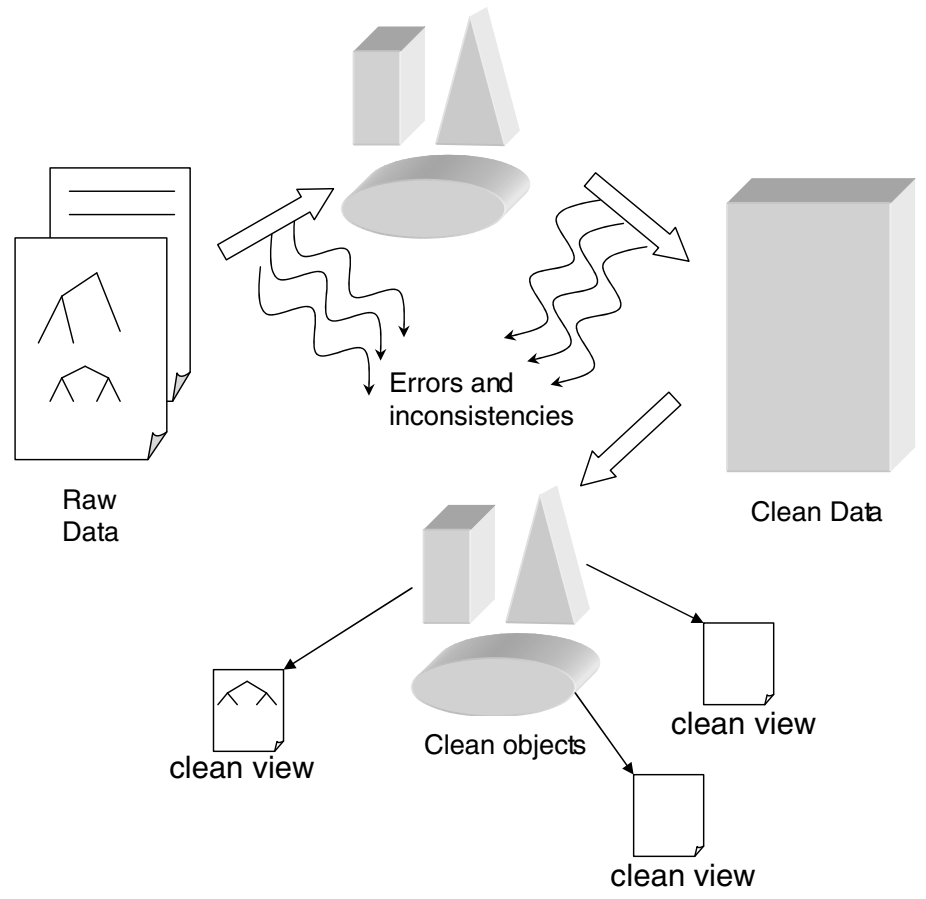

Fig. 3. Interaction lifecycle between the raw data and extracted information. 
the data in the database is stable, most likely a lifecycle would involve reading the database and converting it into objects and performing the required manipulations. This manipulation can be done at three different levels - (i) using generated objects only; (ii) using SQL queries only, (iii) combining both to achieve the simplest data manipulability.

\section{Benefits of This Architecture}

Let's say the user wishes to find an answer to the following question - What is the distribution of named entities that are ARG0s of the predicate "say"? The pseudocode outlined in Fig. 4 shows how you could accomplish that using a combination of SQL and object-level manipulation. Without the current architecture, this manipulation would have required significant pre-processing by each end-user, requiring possible re-interpretation of the underlying semantics of the data itself, and a possible introduction of errors therewith.

While synchronizing and revising the Treebank and PropBank annotations, there were several cases of well-defined changes such as: (i) if an NP representing an ARGM argument, is dominated by a PP, which is dominated by a VP that is the parent of the predicate, then the ARGM label is transferred from the NP to the dominating PP; (ii) if an argument tagged on an NP is dominated by an SBAR, dominated by a WH-phrase, then a separate semantic link (SLINK) is created that links the ARG label on the WH-pronoun to its referent, the latter of which was previously directly tagged as an argument of the predicate; (iii) traces are inserted to address some synchronization issues; (iv) argument attachments, and

Procedure: get_name_entity_distribution(a_arg_type, a_predicate_lemma)

1: Load OntoNotes database

2: for all proposition $\in$ on.proposition.proposition_bank.propositions () do

if proposition.predicate.lemma $==$ a_predicate_lemma then

(arg_type, arg_id) $\leftarrow$ get_arg_info("select $*$ from argument where proposition_id = proposition.id")

for all arg_type $\in$ arg_types do

if arg_type $==$ a_arg_type then

node_id $\leftarrow$ get_arg_node_id("select $*$ from argument_node where argument_id = argument.id")

document_id $\leftarrow$ proposition.document_id

sentence_id $\leftarrow$ proposition.sentence_id

document $\leftarrow$ on.tree.treebank.get_tree_document(document_id)

tree $\leftarrow$ document.get_tree(sentence_id)

node $\leftarrow$ tree.get_subtree(node_id)

name_entity_type $\leftarrow$ node.name_entity

name_entity_hash[name_entity_type] $\leftarrow$ name_entity_hash[name_entity_type] +1 end if

end for

end if

end for

19: return name_entity_hash

Fig. 4. Pseudocode for performing a cross-layer query on the representation using the ontonotes-db-tool API. 
therefore phrase attachments were altered. More of such changes are mentioned in [1]. All these changes required some degree of change to the Treebank, and therefore all the pointers in PropBank annotation had to be revised to be consistent with the Treebank. Since we did not have this tool/representation when we performed these merges, it was quite a painful and kludgy experience. There are tools such as TSurgeon, which do allow arbitrary manipulations of tree structures, but fail to be very useful in such cases where multiple representations are simultaneously affected, and have to be synchronized. In light of this new representation and API, those changes would be extremely easy.

We used the OntoNotes data in the Lexical Sample WSD task of the SemEval competition [15]. It was extremely easy to format the word sense information from OntoNotes to conform to the predetermined Semeval lexical-sample task format.

The amount of coreference annotation in OntoNotes was richer and more in quantity than any other effort in the past. However, the tool that we used was a generic tool called "Callisto"c developed at MITRE. Although it is a very flexible tool, it does not provide mechanisms to add data-level consistency checks based on the semantics of the individual tasks that you use it to accomplish. One recurring manifestation of this limitation was that annotators could erroneously add a coreference link to multiple coreference chains. To correct this, we added a quality control step which comprised of trying to add the annotated documents to the database, and inserted a routine using the DB-API, that created a report of multiple-link errors, which the annotators could easily read, and edit the coreference chains accordingly to eliminate the inconsistencies. This process was repeated until no errors were identified while loading a document to the database.

Even in the well established, and heavily-used Penn Treebank, we identified some orphan, or duplicate traces that were subsequently corrected. This process identified many more such cases in the Chinese Treebank, which are relatively new and unexplored by the community - especially since automatic parsers typically tend to ignore this information and not reproduce it.

Last but not least, annotation tools can be built using this representation as their backend, and tools could be easily written to visualize this complex data fairly easily.

\section{Related Works}

Although this is probably the first attempt at combining so many different layers together, the importance of this goal has already been recognized by the community and attempts have been made to reach a consensus; most notably by the formation of the ISO/TC 37/SC 4 standard by the International Standards Organization. This standard identifies principles and methods for creating, processing and managing language resources [9]. A working group within this standard WG1-1 has been trying to put together a Linguistic Annotation Framework (LAF) that can serve as a basis

${ }^{c}$ http://callisto.mitre.org/ 
for harmonizing existing language resources. However, the bottomline is that this is a framework, and whoever wants to conform to that has to define the schemas and write translation routines to convert their data into the required representation which is a form of feature structure graph. We envelope our specific six layers by efficiently capturing their internal semantics at a skeletal level and provide an object-level representation that could be easily translated into any format that might evolve from this standard.

Another feature structure representation - GLARF [11] tries to capture information in various Treebanks, and also tries to superimpose some predicate argument structure. This technique is more representation centric - trying to capture a union of various individual representations, however, as per our understanding, without any means of easy access to the data. It is also not clear how it would extend to accommodating more layers of semantic information.

An additional significant effort is the Unified Linguistic Annotation (ULA) project [17] which is also a collaborative effort aimed at merging several existing semantic annotation efforts: PropBank, NomBank, Coreference, the Penn Discourse Treebank, and TimeBank. Crucially, all individual annotations are being kept separate in order to make it easy to produce alternative annotations of a specific type of semantic information (e.g., word senses, or anaphora) without needing to modify the annotation at the other levels. One of the main goals of this project is to eliminate any theoretical incompatibilities between the different layers.

In the past, database representations have been used to store individual annotation layers as in the case of FrameNet [2] as well as WordNet. However, they are restricted to predicate argument structures and pure ontological representations, respectively. Neither of them have provided native layers of API for easy manipulation. The distribution is a collection of XML documents, so end-users have to write routines to read and manipulate the data themselves. Recently, as part of the Unstructured Information Management Architecture (UIMA) [8] effort, IBM has introduced a mechanism called Common Analysis System (CAS) [5] that allows definition of annotation types and serialization as well as querying capabilities. To our knowledge, it has not yet been widely used, or reported, so a detailed comparison is not possible at this time.

Two other works that comes closest to ours in terms of the types of annotations and corpora itself, are the Prague Dependency Treebank [6] and the Salsa project $[4,18]$. However, even these do not include word senses, their connections to the Ontology, and a full range of coreference. Neither do they address additional languages (Chinese and Arabic) and genres (Broadcast News, Talk Shows, etc.) that we address.

\section{Conclusions}

In conclusion, we have created a corpus with various levels of semantic information integrated in one big database. This process identified several levels of inconsistencies that were resolved, ensuring a clean, consistent final product. 
The relationships between all the layers and within the layers themselves is efficiently captured in the database schema. We have also provided an object layer on top of the database layer, written in Python, which can flexibly manipulate the data at the level of the database or as objects, to extract information across layers which was not easily possible before. It can also produce the individual layers by themselves as well as a human readable representation. All this is available for distribution through the Linguistic Data Consortium (LDC). This should facilitate defining custom views of the data as well as extracting cross-layer features for use in predictive models, neither of which was easily possible previously.

\section{Acknowledgments}

We gratefully acknowledge the support of the Defense Advanced Research Projects Agency (DARPA/IPTO) under the GALE program, DARPA/CMO Contract No. HR0011-06-C-0022.

\section{References}

[1] Olga Babko-Malaya, Ann Bies, Ann Taylor, Szuting Yi, Martha Palmer, Mitch Marcus, Seth Kulick, and Libin Shen, Issues in synchronizing the English treebank and propbank, in Workshop on Frontiers in Linguistically Annotated Corpora 2006, July 2006.

[2] Collin Baker, Charles Fillmore, and Beau Cronin, The structure of the framenet database, International Journal of Lexicography 16(3) (2003) 281-296.

[3] Collin F. Baker, Charles J. Fillmore, and John B. Lowe, The Berkeley FrameNet project, in Proceedings of the International Conference on Computational Linguistics (COLING/ACL-98), Montreal (1998), ACL, pp. 86-90.

[4] Katrin Erk and Sebastian Pado, A powerful and versatile xml format for representing role-semantic annotation, in Proceedings of LREC, 2004.

[5] T. Gotz and O. Suhre, Design and implementation of the uima common analysis system, IBM Systems Journal 43(3) (2004).

[6] Jan Hajic, B Vidova-Hladka, and P. Pajas, The prague dependency treebank: Annotation structure and support, in Proceeding of the IRCS Workshop on Linguistic Databases (2001), pp. 105-114.

[7] Eduard Hovy, Mitchell Marcus, Martha Palmer, Lance Ramshaw, and Ralph Weischedel, OntoNotes: The $90 \%$ solution, in Proceedings of HLT/NAACL, New York City, USA, June 2006. Association for Computational Linguistics, pp. 57-60.

[8] IBM. Unstructured information management architecture (UIMA) - http://www. research.ibm.com/uima, 2005.

[9] Nancy Ide and Laurent Romary, International standard for a linguistic annotation framework, Journal of Natural Language Engineering 10(3-4) (2004) 211-225.

[10] Mitchell P. Marcus, Beatrice Santorini, and Mary Ann Marcinkiewicz, Building a large annotated corpus of English: The Penn treebank, Computational Linguistics 19(2) (1993) 313-330.

[11] Adam Meyers, R. Grishman, M. Kosaka, and S. Zhao, Covering treebanks with glarf, in ACL/EACL Workshop on Sharing Tools and Resources for Research and Education, 2001.

[12] Martha Palmer, Olga Babko-Malaya, and Hoa Trang Dang, Different sense granularities for different applications, in Robert Porzel, editor, HLT-NAACL 2004 Workshop: 
2nd Workshop on Scalable Natural Language Understanding, Boston, Massachusetts, USA, May 2 - May 7 2004. Association for Computational Linguistics, pp. 49-56.

[13] Martha Palmer, Daniel Gildea, and Paul Kingsbury, The Proposition Bank: An annotated corpus of semantic roles, Computational Linguistics 31(1) (2005) 71-106.

[14] Andrew Philpot, Eduard Hovy, and Pantel Patrick, The omega ontology, in Proceedings of the ONTOLEX Workshop at IJCNLP, Jeju Island, South Korea, October 2005.

[15] Sameer Pradhan, Edward Loper, Dmitriy Dligach, and Martha Palmer, Semeval2007 task-17: English lexical sample, srl and all words, in Proceedings of the Fourth International Workshop on Semantic Evaluations (SemEval-2007), Prague, Czech Republic, June 2007. Association for Computational Linguistics, pp. 87-92.

[16] Sameer Pradhan, Lance Ramshaw, Ralph Weischedel, Jessica MacBride, and Linnea Micciulla, Unrestricted coreference: Indentifying entities and events in ontonotes, in Proceedings of the IEEE International Conference on Semantic Computing (ICSC), September 17-19, 2007.

[17] James Pustejovsky, Adam Meyers, Martha Palmer, and Massimo Poesio, Merging PropBank, NomBank, TimeBank, Penn Discourse Treebank and coreference, in Proceedings of the Workshop on Frontiers in Corpus Annotations II: Pie in the Sky, Ann Arbor, MI, June 2005.

[18] Dennis Spohr, Aljoscha Burchardt, Sebastian Pado, Anette Frank, and Ulrich Heid, Inducing a computational lexicon from a corpus with syntactic and semantic annotation, in Proceedings of IWCS-7, 2007.

[19] Ralph Weischedel and Ada Brunstein, BBN pronoun coreference and entity type corpus LDC catalog no.: LDC2005T33. BBN Technologies, 2005.

[20] L. C. Yu, C. H. Wu, A. Philpot, and E. H. Hovy, Ontonotes: Sense pool verification using google n-gram and statistical tests, in Proceedings of the OntoLex Workshop at the 6th International Semantic Web Conference (ISWC 2007), 2007. 